\title{
DISSOLUTION OF THE RAT ZONA PELLUCIDA BY ACIDIFIED MEDIA AND BLASTOCYST VIABILITY
}

\author{
J. L. BRUN AND A. PSYGHOYOS \\ Centre National de la Recherche Scientifique, \\ E.R. 122-Physiologie de la Reproduction, \\ Hôpital de Bicêtre, I.N.S.E.R.M., \\ 78 Avenue du Général Leclerc, 94-Bicêtre, France
}

(Received 4th April 1972, accepted 27th April 1972)

Exposure of mouse blastocysts in vitro to acidified media effective in dissolving the zona pellucida (Gwatkin, 1964), was reported to be incompatible with the survival of the blastocyst (Bowman \& McLaren, 1969). It has been concluded that decreased $\mathrm{pH}$ is unlikely to be responsible for the lysis of the zona pellucida in utero (Bowman \& McLaren, 1970). This conclusion was drawn from data concerning the effect of a prolonged exposure (up to several hours) of eggs to acidity in vitro. Evidence is reported here to show that the dissolution of the zona pellucida by a brief exposure of the blastocyst to acidity is compatible with its viability and subsequent development.

Solutions of adenosine- 5 triphosphate (disodium salt of ATP, equine muscleSIGMA) were made in $0.9 \%$ saline solution at concentrations of ATP ranging from $0.33 \mu \mathrm{M}$ to $5.4 \mu \mathrm{M}$. The $\mathrm{pH}$ of these solutions was found to be between 5.06 and 3.06 , respectively. A buffer solution, $0.2 \mathrm{~m}$ sodium acetate-acetic acid, $\mathrm{pH} 3 \cdot 7$, was also used to test the effect of this $\mathrm{pH}$ on the zona pellucida. The blastocysts were obtained from adult rats of Wistar strain by flushing the uterine horns with Tyrode. After being rinsed three times in saline, the blastocysts were placed in groups of five, in a watch-glass containing the solution to be tested. The dissolution of the zonae was followed under a light microscope at room temperature $\left(22^{\circ} \mathrm{C}\right)$.

It was found that the exposure time needed for the dissolution depended on the concentration of the ATP and, hence, on the $\mathrm{pH}$. The time for the dissolution

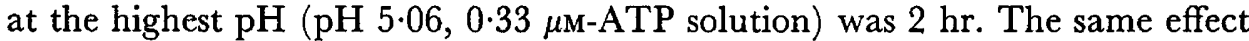
was achieved in 2 min at the lowest $\mathrm{pH}$ (pH 3.36, 5.4 $\mu$ M-ATP) (PI. 1, Fig. 1). The sodium acetate-acetic acid buffer ( $\mathrm{pH} 3.7$ ) was as effective in dissolving the zona as the ATP solution of equivalent acidity. Furthermore when the ATP was dissolved in the buffered medium serving for culture $(\mathrm{pH} \mathrm{7.4)} \mathrm{instead} \mathrm{of} \mathrm{in}$ saline, the zonae were not dissolved. A microdrop of ATP-saline solution $(\mathrm{pH}$ 3.7) was introduced into the medium close to one of the blastocysts. The zona pellucida of this blastocyst instantaneously disintegrated in the region which came into immediate contact with the microdrop, and progressively dissolved for the next $2 \mathrm{~min}$. The dissolution then stopped, probably due to the buffering effect of the rest of the medium, leaving intact a fragment of the zona (Pl. 1, Fig. 2). 
The blastocysts studied for their viability and subsequent development were those which were zona-free following their exposure for $2 \mathrm{~min}$ to the sodium acetate-acetic acid buffer ( $\mathrm{pH} 3.7$ ) or to ATP-saline solution at the same $\mathrm{pH}$. Once the zonae had been dissolved in the test solution, the blastocysts were replaced in Tyrode and were separated into two groups. One group was rinsed three times in the culture medium (Eagle $\mathrm{L}$ medium containing $5 \%$ fetal calf serum-DIFCO) and then placed in adequate culture conditions (BittonCasimiri \& Psychoyos, 1968). During the 5 days of culture, these blastocysts were found to behave in the same way as the normal blastocysts which lose their zona in utero (i.e. those recovered from the uterus in the evening on Day 5 of pregnancy). They all expanded and their outgrowth started 40 to $48 \mathrm{hr}$ after the beginning of their life in vitro.

A second group of blastocysts was introduced on Day 5 of pregnancy into one of the uterine horns of rats in which both tubes were ligated on Day 2 of pregnancy. The contralateral horn of these animals received control blastocysts, i.e. blastocysts not previously treated with acidified media with their zonae intact. A laparotomy was performed 5 days later to examine the implanted embryos. The implantation rate was $19 \%$ for the treated blastocysts $(13 / 54)$ and $48 \%$ for the control ones (26/54). All implanted blastocysts of both groups developed to normal young. The difference in the percentage between control and treated blastocysts should be attributed to technical difficulties in the transfer due to the stickiness of the zona-free blastocysts.

It is evident that a brief exposure of the rat blastocyst to an acidified medium inducing lysis of the zona pellucida in vitro is compatible with egg viability. The possibility that a transient acidosis of the egg environment is involved in the lysis of the zona in utero cannot therefore be excluded. The discreet lowering of $\mathrm{pH}$ which has been reported to occur in the mouse uterine lumen on the day when zona lysis occurs (McLaren, 1970) may reflect a hormone-dependent transient acidosis occurring either once or repeatedly on the luminal surface of the epithelium. At this time, the epithelium surrounds the blastocyst and is in contact with it (Psychoyos, 1966; Potts, 1969; Tachi, Tachi \& Lindner, 1970), the dissolution of the zona could take place in a way similar to that occurring in vitro when a microdrop of an acidified medium is placed in the proximity of the blastocyst. Histochemical studies (Christie, 1966) have shown that in the rat, the transition to the uterine state of receptivity is accompanied by a pronounced ATP-ase activity on the surface of the luminal epithelium. This activity may be linked with the active transport of various factors through the cellular membranes. It may also indicate the occurrence of an ATP dephosphorylation and consequently, a surface ionic change.

\section{EXPLANATION OF PLATE 1}

FIG. 1. Dissolution of the zona pellucida of a 5-day blastocyst placed in a $5.4 \mu \mathrm{M}$-solution of ATP in saline ( $\mathrm{pH} 3 \cdot 36$ ). Within 2 min (photos 1 to 5 ), the zona disintegrated and dissolved. $\times 350$.

FIG. 2. A microdrop of ATP-saline solution (pH 3.7) was introduced at point $x$ into the buffered medium used for culture of a 5-day blastocyst incubated in $1 \mathrm{ml}$ buffered $(\mathrm{pH}$ 7.4 ) culture medium (Eagle L medium containing $5 \%$ fetal calf serum). Within 2 min, the region of the zona exposed to acidity disintegrated and dissolved while the opposite region remained intact (photos 1 to 3 ). $\times 350$. 
PIATE 1
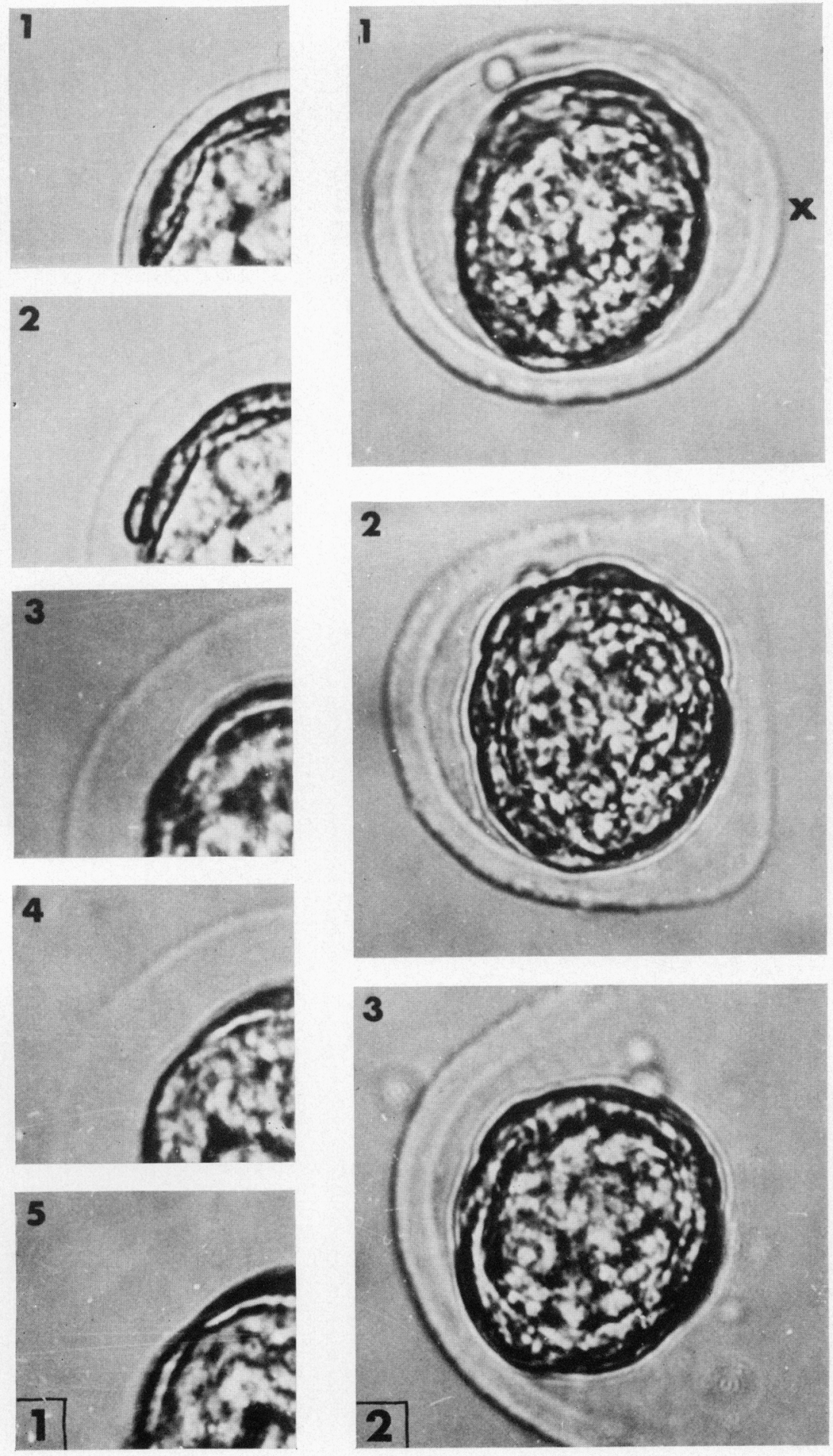

(Facing p. 490) 


\section{REFERENCES}

Bitton-Casimiru, V. \& Psychoyos, A. (1968) Développment du blastocyste du rat in vitro. C.r. hebd. Seanc. Acad. Sci., Paris, 267, 762.

Bowman, P. \& McLaren, A. (1969) The reaction of the mouse blastocyst and its zona pellucida to $\mathrm{pH}$ changes in vitro. . Reprod. Fert. 18, 139.

Bowman, P. \& McLaren, A. (1970) The reaction of the mouse blastocyst and its zona pellucida to enzymes in vitro. F. Embryol. exp. Morph. 24, 331.

Chrustie, G. A. (1966) Implantation of the rat embryo: glycogen and alkaline phosphatases. $\mathcal{F}$. Reprod. Fert. 12, 279.

Gwatkin, R. B. L. (1964) Effect of enzymes and acidity on the zona pellucida of the mouse egg before and after fertilization. 7. Reprod. Fert. 7, 99.

McLAREN, A. (1970) Early embryo-endometrial relationships. In: Ovo-Implantation, Human Gonadotrotropins and Prolactin, p. 18. Ed. Karger, Basel.

Potrs, M. (1969) The ultrastructure of egg implantation. In: Advances in Reproductive Physiology, Vol. 4, p. 241. Ed. A. McLaren. Logos Press, London.

Psychoyos, A. (1966) Recent researches on egg implantation. Ciba Foundation Study. No. 23, p. 4. Churchill, London.

TACHI, S., TACHI, C. \& LindNER, H. R. (1970) Ultrastructural features of blastocyst attachment and trophoblastic invasion in the rat. f. Reprod. Fert. 21, 37. 